mann: ++ * Luetin: -. Kidney Function-first hour 50, second hour 18 , total 68 per cent.

CASE 45.-Female, aged sixty years. Diagnosis-carcinoma of liver and gall-stones. Systolic blood-pressure, 172; diastolic blood-pressure, 90. Family IIistory-negative. Wassermann: -. Luetin: - .

CASE 46.-Male, aged fifty-eight years. Diagnosis-detached retina; hypertension; systolic blood-pressure, 185. Family History -brother at thirty-eight had slight "stroke." He had no known venereal lesion. Personal History--no venereal infection. Wassermann: - Luetin: ++ .

CASE 47.-Female, aged sixty-one years. Diagnosis--aortitis; systolic blood-pressure, 190; diastolic blood-pressure, 85. Family IFistory-husband has angina, though he denies lues. Wassermann -latcr. Luetin: ++. Kidney Function-first hour 39, second hour 10 , total 49 per cent.

CASE 48.-Male, age about fifty years. Diagnosis-aortitis; systolic blood-pressure, 200; diastolic blood-pressure, 100. Personal IIistory-denies infection. Wassermann: ++ . Luetin-not done.

CASE 49.-Female, aged forty-eight years. Diagnosis-hypertension; systolic blood-pressure, 184; diastolic blood-pressure, 84. Personal History-husband a drunkard; suicide. Wassermann: $+{ }^{*}$ Luetin: + .

CASE 50.-Female, aged sixty-four years. Diagnosis-hypertension; systolic blood-pressure, 200+. Family History hypertension for ten ycars; negative. Personal History-negative Wassermann-not done. Luetin: ++ .

\title{
LATENT SYPHILITIC INFECTION OF THE LUNGS.
}

\author{
By H. R. M. Landis, M.D., \\ AND \\ Paul A. Lewis, M.D., \\ PHILADELPHIA.
}

THE only type of pulmonary syphilis about which there is a general agreement is that seen in newborn syphilitic children. In this form of the disease gummata may occur, but, as a rule, they are rare. The typical lesion is that known as white pneumonia. The lung presents on section a dry, smooth surface, grayish or yellowish white in color, resembling closely the cut surface of the pancreas. The process may occur in scattered patches throughout both lungs or may involve the entire lobe. This type of pulmonary syphilis is, for the most part, of pathological interest only. 
In acquired syphilis most writers describe two main types of the disease as it occurs in the lungs, namely, the formation of gummata or an extensive cellular infiltration which leads to fibroid changes. If the disease assumes the indurative type, and this is by far the most common form, it usually originates at the hilus of the lung and extends outward along the bronchi and bloodvessels. The process is usually unilateral, and at most involves only a portion of one lobe; if several lobes are implicated it is the portions which adjoin the root of the lung.

In addition to these types a focal form has been described in which the lesion consists of an area of consolidation and catarrh. It is usually situated around the root of the lung, but may occur at one apex.

Whether the disease manifests itself in the form of gummata as a diffuse fibrosis or as a focal lesion, most of the cases reported indicate that the base of the lung or the area about the hilus, rather than the apex, is the part most frequently attacked. This fact is usually cited as one of the strong differential points between syphilis and tuberculosis. The general opinion has been that the apices are rarcly involved, but it would be rather surprising, in view of the wonderfully diverse forms in which the localization of syphilis manifests itself, if the upper portions of the lungs should always escape.

We believe that pulmonary syphilis of a latent type occurs far more frequently than is usually supposed. The form to which we wish to call attention is that in which the localization occurs in the apices of the lungs. This type of the disease may simulate early pulmonary tuberculosis so closely as to deceive us entirely. How closely the two diseases may resemble each other is illustrated by the following eases:

Case I (Phipps Institute, No. 9485).--Female, aged twentyeight years, first came to the Phipps Institute June 10, 1911. Her first pregnancy had resulted in a miscarriage. She gave a history of having had repeated attacks of blood-spitting for five years. During the two years prior to her coming to the dispensary she had lost thirty pounds in weight, and for a year had had a cough, considerable greenish expectoration, and might sweats. She also complained of pain posteriorly over the base of the right lung. There was a slight elevation of temperature, $99^{\circ}$ to $100^{\circ} \mathrm{F}$.

ExAMination. There was some flattening beneath the right clavicle, diminished expansion, impairment of resonance on percussion, and bronchovesicular breathing.

She was sent to a sanatorium and remained away for several months, where she gained about sixteen pounds in weight, but the pulmonary symptoms remained unchanged. On her return to the Phipps Institute her sputum was repeatedly examined for tubercle bacilli as well as other organisms, but always with a negative result. 
Guinea-pigs inoculated with her sputum were killed after an interval of several months, and showed no evidence of tuberculosis. At the end of ten months her condition, aside from some gain in weight, was about the same as when first seen. A Wassermann test taken at this time was strongly positive. She was then given salvarsan, followed by mixed treatment, which was continued for months. Following the salvarsan the symptoms at once began to improve, and she has apparently made a complete recovery. Three subsequent Wassermann tests have been negative. During the past year she has given birth to a healthy child. At the present time (May 15, 1915) she weighs 188 pounds, and is entirely free from pulmonary symptoms.

Case II (Phipps Institute, No. 9617).--Male, aged thirty-three years, husband of the previous case. First visited the Phipps Institute July 6, 1911, stating that he had had a cough and greenish-colored expectoration for the past two years. He also complained of pain posteriorly over the base of the right lung. $\mathrm{He}$ had lost weight and had a slight elevation of temperature in the afternoon.

Examination. Patient was quite anemic. Expansion was diminished beneath the right clavicle, the percussion note was impaired, and the breathing bronchovesicular, with a harsh, prolonged expiratory sound.

He was also sent to a sanatorium, and remained away for six months. Although he gained forty pounds in weight the pulmonary symptoms persisted, and in addition his sputum was blood-streaked at times. On his return to the Phipps Institute his sputum was repeatedly examined for tubercle bacilli and other organisms and also inoculated into guinea-pigs, but with negative results. A Wassermann test taken about a year from his first visit was found to be strongly positive. The administration of salvarsan and later mixed treatment caused a disappearance of his pulmonary symptoms. At the present time (May 15, 1915) he is apparently in perfect health.

CASE III (Phipps Institute, No. 4050).-Male, aged twentynine years, was first seen in the dispensary of the Phipps Institute April 7, 1906. He came because of loss of weight, dyspnea on exertion, and morning cough and expectoration. On séveral occasions the sputum had been blood-streaked.

The family history was negative. He admitted having had gonorrhea eight years previously, but denied ever having had syphilis.

Examination. When first seen there were fine crackling rales at both apices, but otherwise the pulmonary signs were negative. The heart was normal. The sputum was negative.

This patient was seen at intervals during the next two years, and for a period of two months, in 1908, was treated in the wards 
of the Institute. During his stay in the hospital the temperature chart showed frequent elevations, ranging from $99^{\circ}$ to $99.3^{\circ} \mathrm{F}$. While in the hospital he was given three injections of tuberculin. Following the third injection of $10 \mathrm{mg}$. there was a slight elevation of temperature. While his symptoms and general condition remained unchanged the rales at the apices had disappeared. 'The sputum continued to be negative for tubercle bacilli. On leaving the hospital he returned to the dispensary on several occasions, and then ceased to come. After an interval of four years he again reported March 30, 1915. His weight was the same as when last seen. He still had slight fever, and the morning cough and expectoration persisted. In addition he complained of nocturnal pain in his arms and hands and attacks of substernal pain and dyspnea. He had been married about a year when first seen in 1906 . In the intervening nine years his wife had given birth to six healthy children.

The physical examination at this time showed no abnormality in the lungs. There was, however, a diastolic murmur at the aortic area. Believing this to be syphilitic in character a Wassermann test was made, which proved to be strongly positive. This case is of special interest because of the long duration of the pulmonary symptoms and the latent character of the trouble.

CASE IV (Phipps Institute, No. 13391).-Female, aged thirtyfour years, first visited the Phipps Institute August 20, 1914. Her family history was negative. She had been married eleven years and had had three miscarriages. No living children. There was no history of an initial lesion or of secondaries. She came to the dispensary because of a cough, greenish-colored expectoration, night sweats and some loss in weight. In addition she complained of precordial pain, which had been present intermittently for six years. For four years she had suffered from attacks of burning and tingling in the legs.

Examination. Some flattening beneath the right clavicle, impairment of the percussion note, and bronchovesicular breathing. Heart was negative. The reflexes were exaggerated.

During the first six months she was under observation the night sweats, morning cough, and expectoration persisted, and the sputum was blood-tinged on one occasion. There was also a slight rise in temperature. The sputum was negative for tubercle bacilli. In February, 1915, she first complained of nocturnal headache. This gradually became worse, and finally was so severe as to interfere with her sleep. The Wassermann test was strongly positive. Under mixed treatment the headache was relieved. Later, following the administration of salvarsan, the cough ceased and the amount of expectoration was greatly reduced.

CASE V (seen in consultation).-This patient was a young male who, when first seen, had been gradually losing weight for 
three months; during this time he had a slight, hacking, and unproductive cough. He was anemic, had a slight afternoon rise in temperature, and in addition had marked malaise.

Examination. At the right apex there was slight diminution in expansion, some impairment of the percussion note, and a fecble respiratory murmur.

The case seemed to be clearly one of incipient pulmonary tuberculosis, and he was accordingly advised to spend the winter at a health resort. He returned six months later with his general health improved in every way, but in the course of a few months his symptoms began to return. At this time his physician noted that the sternal end of the right clavicle was enlarged and tender. The Wassermann test was positive. After two doses of salvarsan he had no further trouble, and has remained well during the past three or four years.

It is to be noted that the symptoms in all of these cases were characteristic of pulmonary tuberculosis, namely, morning cough and expectoration, blood-streaked sputum, loss of weight, and a slight elevation of the temperature. In two there was pain resembling that occurring in pleurisy at the base of the right lung. Furthermore, they all had physical signs indicative of incipient tuberculosis.

Some years ago E. G. Janeway ${ }^{1}$ pointed out that when the viscera became involved during the tertiary stage of syphilis there is apt to be a moderate amount of fever, a loss of weight, and night sweats. Furthermore, a careful physical examination will often reveal some other noticeable evidence of syphilis, such as enlargement and tenderness of the ribs, clavicles or tibiæ, or an enlarged or indurated testicle. Warthin ${ }^{2}$ has emphasized the fact that next to the aorta and heart the testes are the most frequent sites of a syphilitic infection. In a pathological study of 36 cases of syphilis occurring in males, the majority of which were latent and unrecognized during life, an orchitis fibrosa was present in 31. ' In the case of women a history of miscarriages is extremely significant.

If the lungs are involved there is, in addition to the symptoms mentioned above, cough, which may be dry and unproductive or accompanied by a moderate amount of greenish or yellowish expectoration. Blood-streaked sputum may also occur. Pain at the base of the right lung is not infrequent and may be misinterpreted; it is usually due to a syphilitic perihepatitis and not to pleural inflammation.

The presence of latent syphilis of the lung is to be suspected if in addition to pulmonary symptoms there are present elsewhere in the body lesions which are in all probability luetic in nature, such 
as a periostitis, orchitis, iritis, or suspicious throat lesions. The recognition of these cases should be relatively easy.

The type of the disease which offers the most difficulty, and which for the most part, escapes detection, is that in which the symptoms are entirely pulmonary and in which there are no associated syphilitic lesions. The 5 cases we have reported were of this type. In 2 (Case I and II) we were unable at any time to detect manifestations of the disease in other parts of the body. In the other three the clinical picture in the beginning was that of tuberculosis, but the subsequent appearance of an aortitis (Case III), of nocturnal headaches (Case IV), of an osteoperiostitis (Case V), and the presence of a positive Wassermann reaction made it apparent that we had to deal with syphilis rather than with tuberculosis. The correctness of this assumption received additional confirmation in view of the fact that the symptoms were relieved as the result of the administration of antisyphilitic treatment.

The diagnosis must be made by exclusion. Thus if the symptoms and physical signs are those characteristic of tuberculosis and the sputum does not contain tubercle bacilli, or the progress of the case differs from that usually encountered in tuberculosis, the possibility of some other exciting cause should be thought of. Not only should the sputum be examined for organisms other than the tubercle bacillus, but in addition a Wassermann test should be made in every doubtful case.

The therapeutic results in these cases have been specially gratifying. In 4 the use of salvarsan was followed by an almost immediate relief of the symptoms. The remaining case has not been seen since the administration of that drug.

It is now recognized that salvarsan is much more effective in some forms of syphilis than it is in others. Skin lesions, for instance, clear up rapidly under the use of salvarsan, and the same seems to be true of pulmonary lesions.

\title{
CERTAIN PHYSICAL SIGNS REFERABLE TO THE DIAPHRAGM AND THEIR IMPORTANCE IN DIAGNOSIS. ${ }^{1}$
}

\author{
By Richard Dexter, M.D., \\ CLEVELAND, OHIO.
}

(From the Medical Departments of Western Reserve University Medical School and of the Cleveland City Hospital.

THE diaphragm, situated as it is in close relation to the intrathoracic viscera and to the upper abdomen, is involved often in

${ }^{1}$ Read before the Mississippi Valley Medical Association at Cincinnati, Ohio, October 27, 1914. 